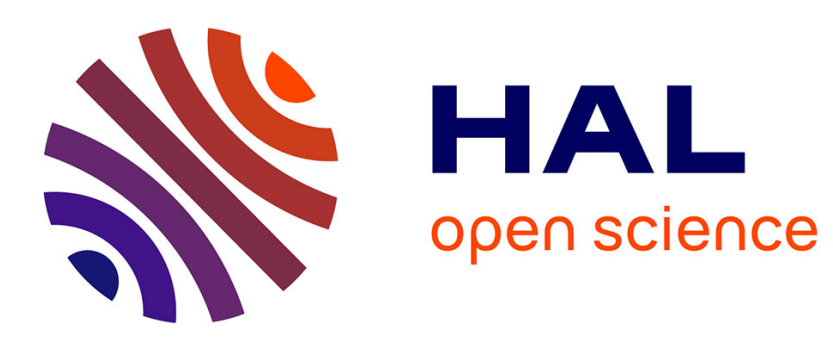

\title{
Control Stability Workspace for a Cable-Driven Parallel Robot Controlled by Visual Servoing
}

Zane Zake, François Chaumette, Nicolò Pedemonte, Stéphane Caro

\section{To cite this version:}

Zane Zake, François Chaumette, Nicolò Pedemonte, Stéphane Caro. Control Stability Workspace for a Cable-Driven Parallel Robot Controlled by Visual Servoing. CableCon 2021 - 5th International Conference on Cable-Driven Parallel Robots, Jul 2021, virtual, France. pp.284-296, 10.1007/978-3030-75789-2_23. hal-03251160

\section{HAL Id: hal-03251160 https://hal.science/hal-03251160}

Submitted on 6 Sep 2021

HAL is a multi-disciplinary open access archive for the deposit and dissemination of scientific research documents, whether they are published or not. The documents may come from teaching and research institutions in France or abroad, or from public or private research centers.
L'archive ouverte pluridisciplinaire HAL, est destinée au dépôt et à la diffusion de documents scientifiques de niveau recherche, publiés ou non, émanant des établissements d'enseignement et de recherche français ou étrangers, des laboratoires publics ou privés. 


\title{
Control Stability Workspace for a Cable-Driven Parallel Robot Controlled by Visual Servoing
}

\author{
Zane Zake $^{1,2}$, François Chaumette ${ }^{3}$, Nicolò Pedemonte ${ }^{2}$, and Stéphane Caro ${ }^{1,4}$ \\ 1 Laboratoire des Sciences du Numérique de Nantes, UMR CNRS 6004, \\ 1, rue de la Noë, 44321 Nantes, France, \\ Zane.Zake@ls2n.fr, \\ 2 IRT Jules Verne, Chemin du Chaffault, 44340, Bouguenais, France \\ nicolo.pedemonte@irt-jules-verne.fr, \\ 3 Inria, Univ Rennes, CNRS, IRISA, Rennes, France, \\ Francois.Chaumette@inria.fr, \\ 4 Centre National de la Recherche Scientifique (CNRS), \\ 1, rue de la Noë, 44321 Nantes, France, \\ stephane.caro@ls 2 n.fr
}

\begin{abstract}
A novel workspace, called Control Stability Workspace (CSW), is proposed and analyzed in this paper. It is a workspace from the control perspective. It allows us to determine whether the closed-loop controller will be able to guide a cable-driven parallel robot (CDPR) to a desired pose, given that there are perturbations present in the system. The effect of the different perturbations on the CSW is analyzed for two spatial CDPRs with a pose-based visual servoing (PBVS) controller. Indeed, this workspace is not only robot architecture-dependent, but also controldependent. It was found that the model errors, that is, the errors in the cable exit and anchor point coordinates, have the most significant effect on the CSW. However, if the pulleys are small enough, then neglecting their geometry does not lead to a significant reduction of the workspace.
\end{abstract}

Keywords: cable-driven parallel robots · visual servoing · stability . workspace

\section{Introduction}

Cable-driven parallel robots (CDPRs) are characterized by a large workspace. However, knowing this workspace is not a trivial task. A workspace consists of all the CDPR moving-platform (MP) poses, in which certain conditions are fulfilled. Depending on the selected conditions multiple workspaces can be determined $[1,2,3]$. The simplest one is the Static Feasible Workspace (SFW), where the static equilibrium of the MP is used as the condition for defining the workspace $[1,3]$. The Wrench Feasible Workspace (WFW) is based on the static equilibrium along with the condition that the MP is able to withstand a set of external wrenches, named Required Wrench Set (RWS), while staying in equilibrium. SFW is a special case of WFW when the external wrench is equal to zero and the static equilibrium is achieved by compensating only the MP weight. 
Cable-cable interferences can affect the pose the MP reaches. Indeed, as the cable is no longer a straight line, but instead is bent at the interference point, the model no longer corresponds to the real CDPR and thus a different MP pose is obtained. To avoid such situations one can compute the Interference Free Workspace (IFW), which is the set of all MP poses without collisions between cables $[4,5]$. An extension of this workspace is the Collision Free Workspace (CFW), which is the set of all MP poses without collisions between the cables, the MP, and the environment.

For some tasks a CDPR will be required to attain high velocities and thus a Twist Feasible Workspace should be used [6]. When the dynamics of the robot need to be taken into account, the dynamic workspace needs to be considered. For example, the Dynamic Feasible Workspace (DFW) is the set of dynamic feasible MP poses. Here, a pose is dynamic feasible if the prescribed MP acceleration set is feasible, while cable tensions are kept within their bounds. For planar CDPRs the dynamic equations were solved analytically and thus the boundary of DFW could be found [7]. However, the same strategy cannot be applied to spatial CDPRs due to their increased complexity. For this reason a new workspace called Improved DFW is defined in [8].

All of the mentioned workspaces portray the physical ability of the MP to arrive at a certain pose given a set of conditions that need to be fulfilled. The control of the CDPR is not taken into account, meaning that there is no knowledge whether the controller will be able to guide the MP to the desired pose. For a closed-loop control, such as visual servoing, one can write the closed-loop equation and analyze the system stability with respect to perturbations $[9,10,11]$. Based on this analysis it was then possible to briefly introduce a novel workspace called Control Stability Workspace (CSW) [11]. It allows us to determine whether the controller will be able to guide the MP to the desired pose, given that there are perturbations present in the system. In this paper we make a thorough analysis of the sensitivity of this workspace with respect to perturbations in different parameters. Furthermore, the CSW is determined for two different CDPRs, and thus conclusions are made about the scalability of perturbation ranges as a function of the CDPR size.

\section{Visual Servoing of a CDPR}

A generic control scheme for visual servoing of a CDPR is shown in Fig. $1[9,10,11]$. Fig. 2 illustrates the main components of a CDPR and the frames of interest.

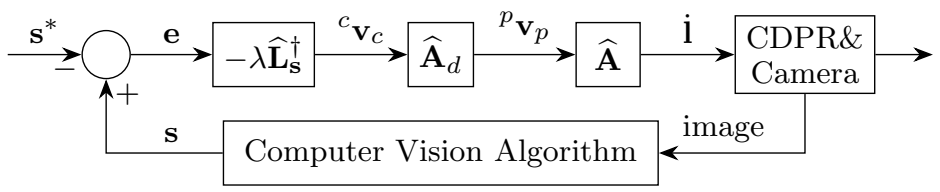

Fig. 1: Control scheme for visual servoing of a CDPR 


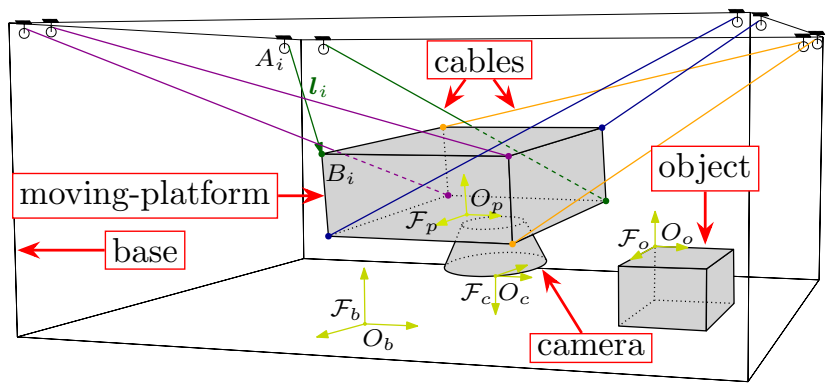

Fig. 2: Schematic of a spatial CDPR with eight cables, a camera mounted on the MP and an object in the workspace

The camera is mounted on the MP in the eye-in-hand configuration. Comparing the current feature vector $\mathbf{s}$, which is available from a computer vision algorithm, to the desired feature vector $\mathbf{s}^{*}$, which is previously defined, it is possible to compute the desired cable velocities that will try to make $\mathbf{s}$ converge to $\mathbf{s}^{*}$. Note that the content of the feature vector $\mathbf{s}$ depends on the chosen visual servoing approach. In case of Pose-Based Visual Servoing (PBVS), we can define $\mathbf{s}=\left({ }^{c} \mathbf{t}_{o}, \theta \mathbf{u}\right)$, where ${ }^{c} \mathbf{t}_{o}$ is the object position in the camera frame $\mathcal{F}_{c}$, and $\theta \mathbf{u}$ is the axis-angle representation of the rotation matrix ${ }^{c *} \mathbf{R}_{c}$ between the current and the desired camera frames [12].

An error $\mathbf{e}$ is computed by comparing $\mathbf{s}$ and $\mathbf{s}^{*}$. To ensure that this error converges to zero, we select as expected behavior:

$$
\dot{\mathbf{e}}=-\lambda \mathbf{e}
$$

where $\lambda$ is a positive gain.

Upon derivation of $\mathbf{e}=\mathbf{s}-\mathbf{s}^{*}$ with respect to time, we get:

$$
\dot{\mathbf{e}}=\mathbf{L}_{\mathbf{s}}^{c} \mathbf{v}_{c}
$$

where $\mathbf{L}_{\mathbf{s}}$ is the interaction matrix and its composition depends on the chosen visual features [12]; ${ }^{c} \mathbf{v}_{c}$ is the velocity of the camera with respect to the base frame $\mathcal{F}_{b}$, expressed in the camera frame $\mathcal{F}_{c}$.

It is then possible to express the camera velocity from (1) and (2):

$$
{ }^{c} \mathbf{v}_{c}=-\lambda \widehat{\mathbf{L}}_{\mathbf{s}}^{\dagger} \mathbf{e}
$$

where $\widehat{\mathbf{L}}_{\mathbf{s}}^{\dagger}$ is the pseudo-inverse of the interaction matrix estimation.

The relation between the camera velocity ${ }^{c} \mathbf{v}_{c}$ and the MP velocity ${ }^{p} \mathbf{v}_{p}$ is expressed as:

$$
{ }^{p} \mathbf{v}_{p}=\mathbf{A}_{d}{ }^{c} \mathbf{v}_{c}
$$

where $\mathbf{A}_{d}$ is the Adjoint matrix [13]:

$$
\mathbf{A}_{d}=\left[\begin{array}{cc}
{ }^{p} \mathbf{R}_{c}\left[{ }^{p} \mathbf{t}_{c}\right] \times{ }_{\times}^{p} \mathbf{R}_{c} \\
\mathbf{0}_{3} & { }^{p} \mathbf{R}_{c}
\end{array}\right]
$$


where ${ }^{p} \mathbf{t}_{c}$ and ${ }^{p} \mathbf{R}_{c}$ are the translation vector and rotation matrix that form the homogeneous transformation matrix ${ }^{p} \mathbf{T}_{c}$ from $\mathcal{F}_{p}$ to $\mathcal{F}_{c}$.

The cable velocity vector $\mathbf{i}$ is computed from MP twist ${ }^{p} \mathbf{v}_{p}$ as follows:

$$
\mathrm{i}=\widehat{\mathbf{A}}^{p} \mathbf{v}_{p}
$$

where $\widehat{\mathbf{A}}$ is the estimation of the Forward Jacobian matrix A. For the modeling, the cables are supposed to be non-elastic and massless. The pulleys are assumed to be small, thus their radius is neglected. The components of matrix $\mathbf{A}$ can thus be expressed in the MP frame $\mathcal{F}_{p}$ as [14]:

$$
\mathbf{A}=\left[\begin{array}{cc}
{ }^{p} \mathbf{u}_{1}^{T} & \left({ }^{p} \mathbf{b}_{1} \times{ }^{p} \mathbf{u}_{1}\right)^{T} \\
\vdots & \vdots \\
{ }^{p} \mathbf{u}_{m}^{T} & \left({ }^{p} \mathbf{b}_{m} \times{ }^{p} \mathbf{u}_{m}\right)^{T}
\end{array}\right]
$$

where ${ }^{p} \mathbf{u}_{i}$ is the unit vector of ${ }^{p} \overrightarrow{A_{i} B_{i}}$, pointing from the exit point $A_{i}$ to the anchor point $B_{i}$ of the $i$ th cable, and ${ }^{p} \mathbf{b}_{i}$ is the vector pointing from the origin of MP frame $\mathcal{F}_{P}$ to $B_{i}$.

The model of the system shown in Fig. 1 is written by injecting Eqs. (3), (4) and (6) into (2):

$$
\dot{\mathbf{e}}=\mathbf{L}_{\mathbf{s}} \mathbf{A}_{d}^{-1} \mathbf{A}^{\dagger} \dot{\mathbf{l}}
$$

The output of the control scheme, that is, the cable velocity vector $\mathrm{i}$, is expressed by injecting (3) and (4) into (6):

$$
\mathrm{i}=-\lambda \widehat{\mathbf{A}} \widehat{\mathbf{A}}_{d} \widehat{\mathbf{L}}_{\mathbf{s}}^{\dagger} \mathbf{e}
$$

where $\widehat{\mathbf{A}}_{d}$ is the estimation of the Adjoint matrix $\mathbf{A}_{d}$.

The closed-loop equation of the system is expressed by injecting (9) into (8):

$$
\dot{\mathbf{e}}=-\lambda \mathbf{L}_{\mathbf{s}} \mathbf{A}_{d}^{-1} \mathbf{A}^{\dagger} \widehat{\mathbf{A}} \widehat{\mathbf{A}}_{d} \widehat{\mathbf{L}}_{\mathbf{s}}^{\dagger} \mathbf{e}
$$

The stability criterion $\boldsymbol{\Pi}$ is defined as:

$$
\boldsymbol{\Pi}=\mathbf{L}_{\mathbf{s}} \mathbf{A}_{d}^{-1} \mathbf{A}^{\dagger} \widehat{\mathbf{A}} \widehat{\mathbf{A}}_{d} \widehat{\mathbf{L}}_{\mathbf{s}}^{\dagger}
$$

According to Lyapunov stability analysis [15], $\boldsymbol{\Pi}>\mathbf{0}$ is a sufficient condition to obtain global asymptotic stability. Indeed, as long as the matrix $\boldsymbol{\Pi}$ is positive definite, it is clear from (10) that the error e will converge to $\mathbf{0 .}$

\section{Control Stability Workspace}

Before using a CDPR, one needs to know its workspace. As recalled in the introduction, many workspace types have been defined [1,2]. For example, the Static-Feasible Workspace (SFW) is defined as [16]:

$$
\mathcal{S}=\left\{\mathbf{p}_{p} \in S E(3): \exists \boldsymbol{\tau} \in \mathcal{T}, \mathbf{W} \boldsymbol{\tau}+\mathbf{w}_{g}=\mathbf{0}_{6}\right\}
$$


Namely, the workspace $\mathcal{S}$ is the set of all MP poses $\mathbf{p}_{p}$ for which there exists a vector of cable tensions $\boldsymbol{\tau}$ within the cable tension space $\mathcal{T}$ such that the CDPR can balance the gravity wrench $\mathbf{w}_{g}$, and $\mathbf{W} \boldsymbol{\tau}+\mathbf{w}_{g}=\mathbf{0}_{6}$. The tension space $\mathcal{T}$ is an $m$-dimensional box of feasible tensions $\mathcal{T}=\left\{\boldsymbol{\tau} \in \mathbb{R}^{m}: \boldsymbol{\tau}_{l b} \leq \boldsymbol{\tau} \leq \boldsymbol{\tau}_{u b}\right\}$ where $\boldsymbol{\tau}_{l b}$ and $\boldsymbol{\tau}_{u b}$ are the lower and upper tension bounds, respectively [17].

SFW is a kineto-static workspace that contains all the poses that the MP is physically able to attain.

From (11) it can be seen that the stability of the system depends on the MP pose, because it shows up in the stability criterion $\Pi$ through the Jacobian matrix $\mathbf{A}$, which is a function of the MP pose. Accordingly, a novel workspace, named Control Stability Workspace (CSW), can be defined as follows [11]:

$$
\mathcal{Z}=\left\{\mathbf{p}_{p} \in S E(3): \forall \mathbf{d} \in \mathcal{D}, \boldsymbol{\Pi}>\mathbf{0}\right\}
$$

The workspace $\mathcal{Z}$ is the set of all MP poses $\mathbf{p}_{p}$, for which the stability criterion $\boldsymbol{\Pi}$ is positive definite for any vector of perturbations $\mathbf{d}$ that is within bounds $\mathcal{D}$. It means that for any MP pose within its CSW, the robot controller will be able to guide the MP to its goal.

\subsection{The Definition of Perturbation Bounds $\mathcal{D}$}

As was shown in $[9,10]$, many parameters can induce perturbations. Furthermore, the perturbations affect the stability of the system simultaneously. To be able to analyze the effect of the perturbations on the CSW, we must first define the perturbation bounds.

Parameters can be either translational or rotational. For example, if we consider the camera pose in the MP frame $\mathcal{F}_{p}$, then the translational parameter is the camera position ${ }^{p} \mathbf{t}_{c}$ and the rotational parameter is the camera orientation ${ }^{p} \mathbf{R}_{c}$ expressed as the axis-angle.

Given a translational parameter $T$, the translational perturbation bound is defined as the radius $r_{t}$ of the sphere shown in Fig. 3. It signifies that the perturbation $\boldsymbol{d}_{t}$ of parameter $T$ that is within the range $\mathcal{D}_{t}$ can have a magnitude of up to $r_{t}$ and can be in any direction. Thus, the perturbed value of parameter $T$ can be anywhere within the sphere, e.g. it could take the value $T_{d 1}$ or $T_{d 2}$.

For the rotational parameter the axis-angle representation is used. The rotational perturbation bound is defined as the angle $\Delta \theta$ for all axis directions $\mathbf{u}$.

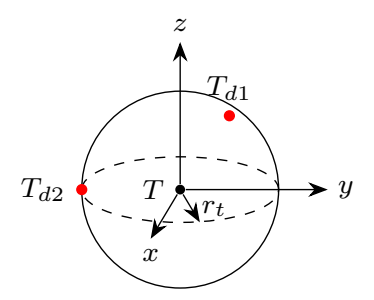

Fig. 3: Translational parameter $T$ and its perturbation radius $r_{t}$ 
That is a perturbation bound $\mathcal{D}_{\theta}$ contains all rotational perturbations with the angle less than or equal to $\Delta \theta$ and any axis $\mathbf{u}$.

\section{Case Study}

\subsection{CDPR prototypes}

As can be seen from (11), the stability criterion $\Pi$ depends on the Jacobian matrix $\mathbf{A}$ and thus we need to consider the CDPR for whom the CSW will be obtained. Here, we use two suspended CDPRs with eight cables, named ACROBOT and CAROCA, shown in Fig. 4. ACROBOT is a $1.2 \mathrm{~m} \times 1.2 \mathrm{~m} \times 1.2 \mathrm{~m}$ cube with a MP size $0.18 \mathrm{~m} \times 0.17 \mathrm{~m} \times 0.07 \mathrm{~m}$ and its mass is $3.5 \mathrm{~kg}$. The dimensions of CAROCA are $7 \mathrm{~m} \times 4 \mathrm{~m} \times 3 \mathrm{~m}$, its MP is $0.42 \mathrm{~m} \times 0.32 \mathrm{~m} \times 0.23 \mathrm{~m}$ and its mass is $150 \mathrm{~kg}$. For these CDPRs we define the baseline perturbation bounds $\mathcal{D}_{b}=\left(r_{b p}, \Delta \theta_{b p}, r_{p c}, \Delta \theta_{p c}, r_{A i}, r_{B i}\right)$ with the following values:

- MP position error range $r_{b p}=0.03 \mathrm{~m}$

- MP orientation error range $\Delta \theta_{b p}=3^{\circ}$

- camera position error range $r_{p c}=0.01 \mathrm{~m}$

- camera orientation error range $\Delta \theta_{p c}=3^{\circ}$

- cable exit point error range $r_{A i}=0.005 \mathrm{~m}$

- cable anchor point error range $r_{B i}=0.005 \mathrm{~m}$

These values are chosen based on the geometry of ACROBOT and taking into account some manufacturing errors that could be present on the robot. For example, there are pulley sheaves of $9 \mathrm{~mm}$ diameter on cable exit points that are not taken into account in the kinematic modeling of the CDPR. Similarly, cable anchor points are modeled as points, while they are actually located on a sphere of $0.008 \mathrm{~m}$ diameter about the modeled points. Camera position and orientation

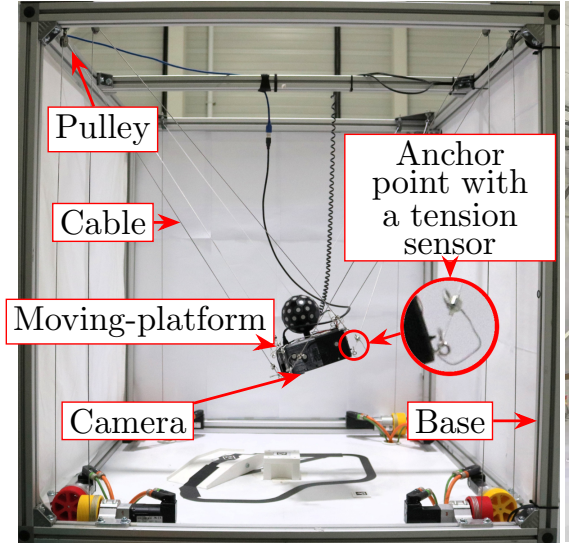

(a)

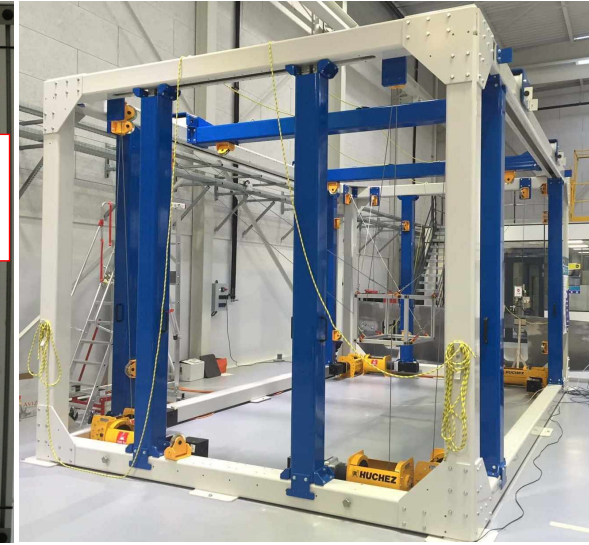

(b)

Fig. 4: CDPR prototypes at IRT Jules Verne: (a) ACROBOT; (b) CAROCA 
errors can also be amounted to manufacturing errors. Finally, the MP position and orientation errors illustrate the possible coarseness of estimation of the MP pose. Indeed, for many CDPRs the initial or homing MP pose is often measured by hand and thus having a small error on the pose estimation is clearly possible. $\mathcal{D}_{b}$ is kept the same for CAROCA to simplify the comparison of results. However, for the supplementary perturbations that will be added to $\mathcal{D}_{b}$, we will consider the particular size of each robot.

\subsection{CSW for ACROBOT with PBVS}

For ACROBOT, MP positions are assigned as follows. The potential workspace is discretized to a set of 12 planes that are parallel to the global $\boldsymbol{x} \boldsymbol{y}$ plane. Each plane is then discretized to a matrix of $33 \times 33$ points with $\mathrm{X}$ and $\mathrm{Y}$ coordinates ranging from $-0.48 \mathrm{~m}$ to $0.48 \mathrm{~m}$ and the step being $0.03 \mathrm{~m}$. The $\mathrm{Z}$ coordinate of the planes ranges from $0 \mathrm{~m}$ to $1.1 \mathrm{~m}$ with the step being $0.1 \mathrm{~m}$. Thus, the full workspace volume is $1.014 \mathrm{~m}^{3}$. The evolution of the workspace volume is shown in Fig. 5. Note that the vertical axis denotes the ratio of the current volume over the maximum volume of $1.014 \mathrm{~m}^{3}$. The CSW is defined in (13) for all MP poses. Thus, at each position multiple MP orientations $\theta \mathbf{u}$ are tested with a maximum angle of $\theta=45^{\circ}$ about any unit axis $\mathbf{u}$. If $\boldsymbol{\Pi}>\mathbf{0}$ for all $\mathrm{MP}$ orientations associated to a given position, then that $\mathrm{MP}$ position is added to the CSW.

First, the workspace corresponding to baseline perturbation bounds $\mathcal{D}_{b}$ is computed. It is shown in Fig. 6(a) and its volume is $0.88 \mathrm{~m}^{3}$, namely, $87.0 \%$ of the full workspace volume. Then the perturbation ranges are incrementally increased one by one, leaving the rest of perturbations as defined in $\mathcal{D}_{b}$. Some examples are shown in Fig. 6. It can be seen that all workspace reduction occurs in a downward direction along $\boldsymbol{z}$ axis no matter the perturbation. This is because ACROBOT is a suspended CDPR and its most stable position is ${ }^{b} \mathbf{t}_{p}=\left[\begin{array}{lll}0 & 0 & 0\end{array}\right]^{\top}$. Indeed, in Fig. 6(i) the tiny workspace is centered around ${ }^{b} \mathbf{t}_{p}=\left[\begin{array}{lll}0 & 0 & 0\end{array}\right]^{\top}$ with about $0.1 \mathrm{~m}$ displacement possible in any direction.

It can be observed that some perturbations have a higher impact on the CSW size than others. Indeed, increasing camera position error range $r_{p c}$ ex-

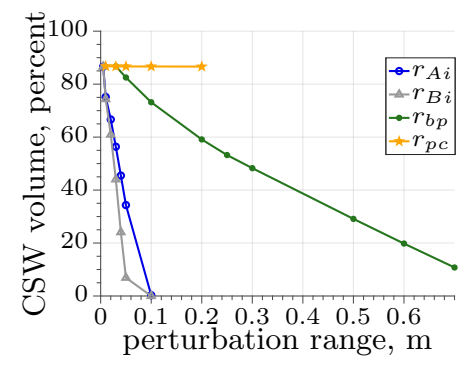

(a)

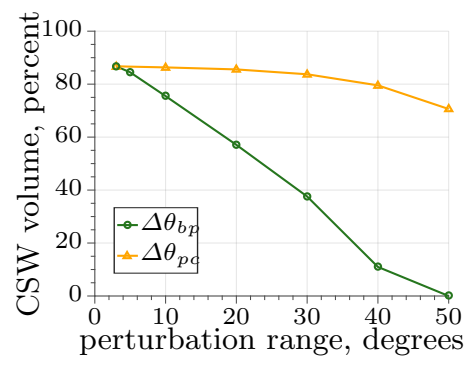

(b)

Fig. 5: CSW volume as a function of perturbation range, ACROBOT with PBVS 


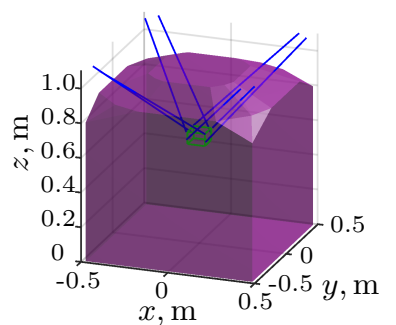

(a) $\mathcal{D}_{b}$

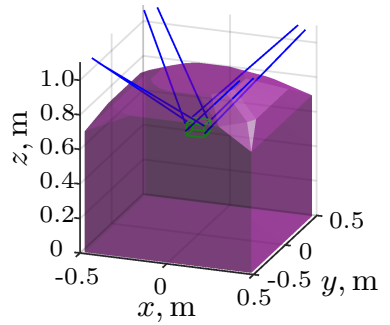

(d) $\Delta \theta_{b p}=10^{\circ}$

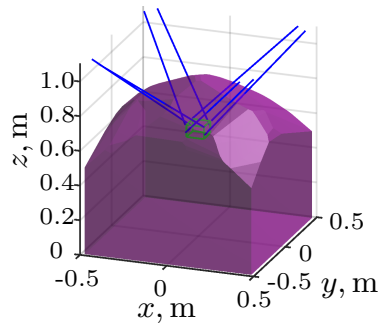

(g) $r_{A i}=0.02 \mathrm{~m}$

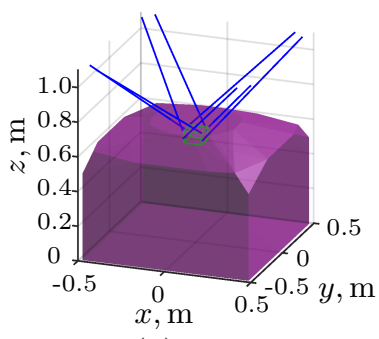

(b) $r_{b p}=0.2 \mathrm{~m}$

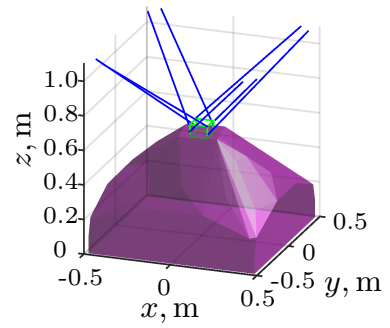

(e) $\Delta \theta_{b p}=30^{\circ}$

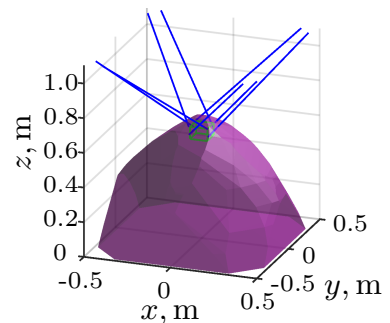

(h) $r_{A i}=0.05 \mathrm{~m}$

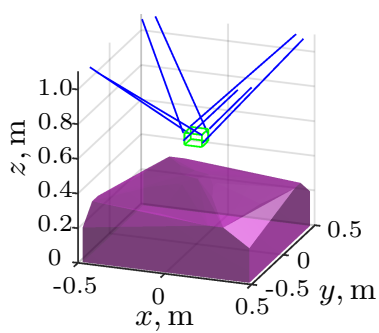

(c) $r_{b p}=0.5 \mathrm{~m}$

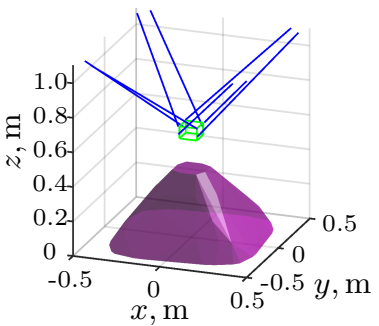

(f) $\Delta \theta_{b p}=40^{\circ}$

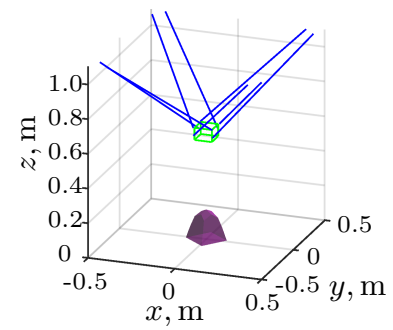

(i) $r_{A i}=0.1 \mathrm{~m}$
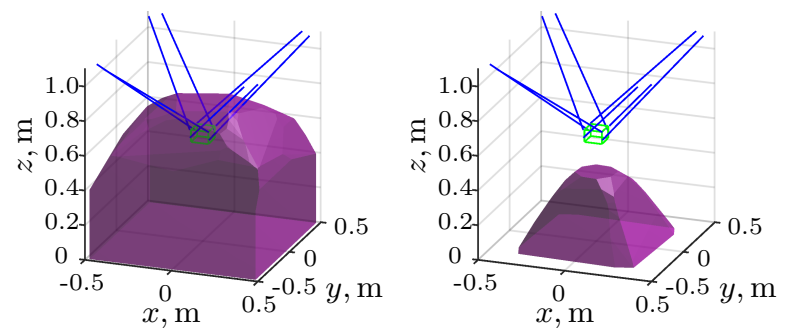

(j) $r_{B i}=0.02 \mathrm{~m}$

(k) $r_{B i}=0.05 \mathrm{~m}$

Fig. 6: Visualization of CSW for ACROBOT with PBVS

pressed in $\mathcal{F}_{p}$ from $0.01 \mathrm{~m}$ to $0.20 \mathrm{~m}$, only slightly decreases the workspace volume from $0.880 \mathrm{~m}^{3}$ to $0.878 \mathrm{~m}^{3}$, as shown in Fig. $5(\mathrm{a})$. Indeed, only $0.4 \%$ of workspace is lost by increasing $r_{p c}$ twenty times. Furthermore, considering the MP size it means that the camera can be anywhere on the MP, without knowing where it is. On the contrary, coarse MP position estimation leads to a steady reduction of CSW, as can be seen in Fig. 5(a). For example, as shown in Fig. 6(b), with 
$r_{b p}=0.2 \mathrm{~m}, 59.1 \%$ of the workspace is still available. Of course this is only true if the other perturbations are kept within the limits defined by $\mathcal{D}_{b}$.

Regarding rotational errors, the results are similar. That is, increasing the MP rotational error $\Delta \theta_{b p}$ has a worse effect on the workspace area than the rotational error on camera pose $\Delta \theta_{p c}$, as can be seen in Fig. 5 (b).

The errors in the CDPR model, namely in cable anchor and exit point coordinates have the highest influence on the CSW volume, as can be seen in Figs. 5(a) and 6. For example, for $r_{B i}=0.02 \mathrm{~m}$, CSW volume is $0.618 \mathrm{~m}^{3}$ (shown in Fig. 6(j)), while for $r_{B i}=0.05 \mathrm{~m}$ the workspace reduces to $0.07 \mathrm{~m}^{3}$ (shown in Fig. 6(k)). Similarly, when $r_{A i}$ is increased, the CSW volume decreases rapidly (shown in Figs 6(g) to 6(i)). However, this decrease is slower than for cable anchor point coordinate errors. Note that, if the error is no more than $0.01 \mathrm{~m}$, then the workspace remains sufficiently large. For ACROBOT, the pulley sheave radius is $0.0045 \mathrm{~m}$ and thus is lower than $r_{A i}=0.005 \mathrm{~m}$ defined for $\mathcal{D}_{b}$. Thus, it is clear that for ACROBOT, the pulleys are small enough to be negligible.

\subsection{CSW for CAROCA with PBVS}

For CAROCA, the potential workspace is discretized into 11 planes with $33 \times 33$ points per plane, just like for ACROBOT. However, here the step between the points and planes is larger. The full workspace volume is equal to $82.33 \mathrm{~m}^{3}$.

The CSW computed for CAROCA with baseline perturbation bounds $\mathcal{D}_{b}$ has a volume of $63.06 \mathrm{~m}^{3}$, which is $76.6 \%$ of the full workspace. The change of the CSW volume depending on perturbation range is shown in Fig. 7, while some examples are shown in Fig. 8. Note that in Fig. 7 the percentage shown is the obtained volume for a given perturbation set over the maximum volume of $82.33 \mathrm{~m}^{3}$. In general, the shape of the curves in Fig. 7 is similar to those in Fig. 5 for ACROBOT. More precisely, perturbation in CDPR model has the highest effect on CSW volume, following by the MP pose estimation and finishing with hand-eye calibration errors. However, in Fig. 8(a) the green curve of the translational error $r_{b p}$ has an exponential shape. Furthermore, with the increased CDPR size, the range of this perturbation has increased. Even having set $r_{b p}=$

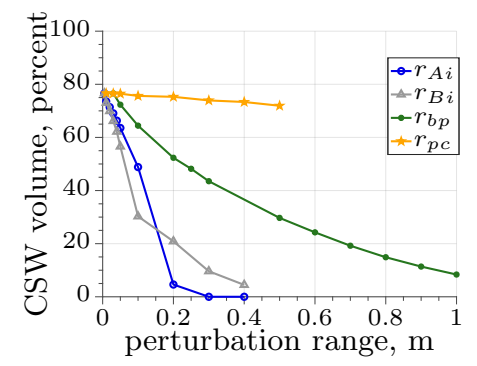

(a)

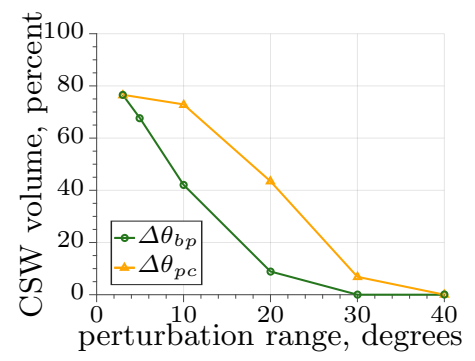

(b)

Fig. 7: CSW volume as a function of perturbation range, CAROCA with PBVS 
$1 \mathrm{~m}$ does not make the workspace null, instead it is equal to approximately $10 \%$ of the full workspace and appears to be almost flat, as can be seen in Fig. 8(d). Similarly, having $r_{B i}=0.1 \mathrm{~m}$ or $r_{A i}=0.1 \mathrm{~m}$ does not make the CSW vanish for CAROCA. Instead, approximately $30 \%$ and $50 \%$ of the full workspace remains available, respectively, as can be seen in Figs $8(\mathrm{j})$ and 8(l). Furthermore, even setting the camera position error range $r_{p c}=0.5 \mathrm{~m}$ has little effect on the CSW size, which remains at $71.9 \%$. On the other hand, rotational errors rapidly reduce the workspace. Especially those in the MP pose estimation, where at $\Delta \theta_{b p}=10^{\circ}$ the volume is at $42.1 \%$ (Fig. $8(\mathrm{e})$ ) and at $\Delta \theta_{b p}=30^{\circ}$ the volume is only at $0.003 \%$ (Fig. $8(\mathrm{f})$ ). While the rotational error $\Delta \theta_{p c}$ on the camera pose also has a significant effect on the CSW size, however having $\Delta \theta_{p c}=30^{\circ}$ allows us to use $43.4 \%$ of the workspace (Fig. $8(\mathrm{~h})$ ), which is significantly larger than for $\Delta \theta_{b p}$ of the same size.

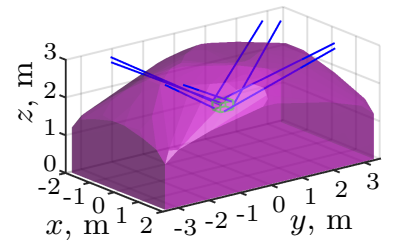

(a) $\mathcal{D}_{b}$

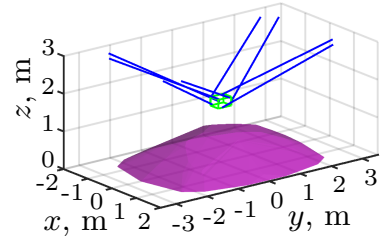

(d) $r_{b p}=1.0 \mathrm{~m}$

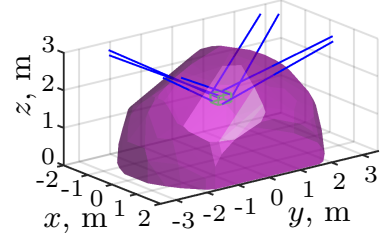

(g) $\Delta \theta_{p c}=20^{\circ}$

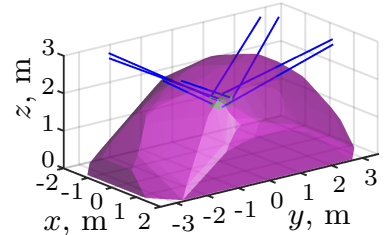

(j) $r_{A i}=0.1 \mathrm{~m}$

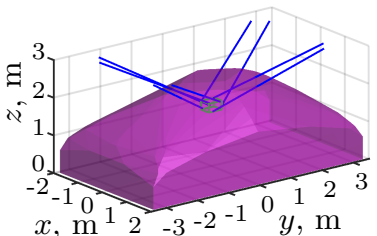

(b) $r_{b p}=0.2 \mathrm{~m}$

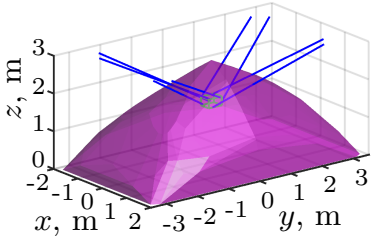

(e) $\Delta \theta_{b p}=10^{\circ}$

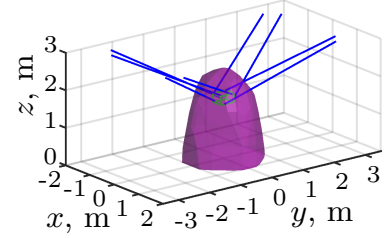

(h) $\Delta \theta_{p c}=30^{\circ}$

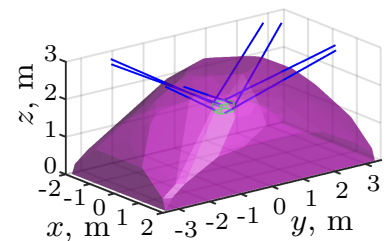

(k) $r_{B i}=0.05 \mathrm{~m}$

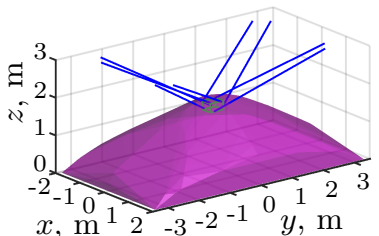

(c) $r_{b p}=0.5 \mathrm{~m}$

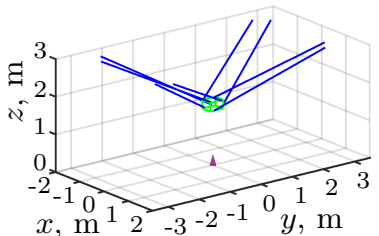

(f) $\Delta \theta_{b p}=30^{\circ}$

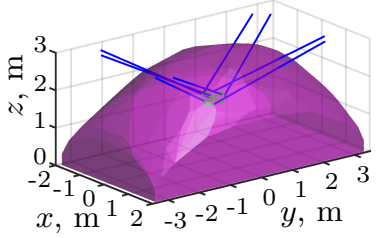

(i) $r_{A i}=0.05 \mathrm{~m}$

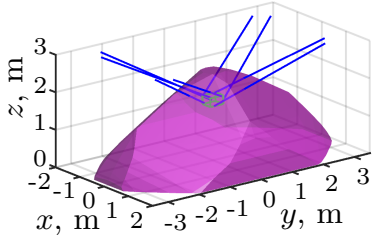

(l) $r_{B i}=0.1 \mathrm{~m}$

Fig. 8: Visualization of CSW for CAROCA with PBVS 
Having such a large CDPR also allows us to compare the change of CSW shape when some perturbations are increased. For example, the increase of $r_{b p}$ is shown in Figs. 8(a)-8(d) and appears to flatten the workspace by greatly reducing the range along $\boldsymbol{z}$ axis before affecting the workspace range in $\boldsymbol{x} \boldsymbol{y}$ plane. On the other hand, rotational errors in hand-eye calibration appear to affect the $\boldsymbol{x} \boldsymbol{y}$ range more severely. This can be seen by comparing Fig. 8(a) with $8(\mathrm{~g})$ and $8(\mathrm{~h})$.

\subsection{Discussion}

Having computed the CSW for two different CDPRs we can conclude that:

- A perturbation on camera position in the MP frame has little to no effect on CSW volume and system stability;

- Similarly, a perturbation on camera orientation in the MP frame a smaller effect than the same perturbation on the MP orientation in the base frame;

- There can be a significant perturbation on the MP position in the base frame without making the system unstable;

- Perturbations on the CDPR model, that is on cable exit and anchor point coordinates, have the largest effect on CSW volume;

- A perturbation on cable anchor points leads to a smaller CSW than same perturbation on cable exit points;

- Translational perturbation bounds scale with CDPR geometry:

$\diamond$ The larger the MP, the larger $r_{B i}$ without making the workspace vanish;

$\diamond$ The larger the CDPR, the larger $r_{A i}$ and $r_{b p}$ can be without making the workspace vanish;

- On the other hand, the rotational perturbations $\Delta \theta_{b p}$ and $\Delta \theta_{p c}$ do not scale with CDPR geometry. They remain approximately the same for ACROBOT and CAROCA.

Note that the obtained CSW is conservative because the perturbation ranges for each parameter were tested in many directions. Thus, the obtained CSW is conservative, because it is the workspace where $\boldsymbol{\Pi}>\mathbf{0}$ for any perturbation direction. An additional study could be done with a fixed set of perturbation ranges, such as $\mathcal{D}_{b}$, to find perturbation direction combinations leading to the largest or to the smallest CSW size.

\section{Conclusions}

A novel workspace, named Control Stability Workspace (CSW), was analyzed in this paper. It is a workspace from the control perspective that allows us to determine whether the controller will be able to guide the MP of the CDPR to the desired pose in the presence of perturbations. The workspace was computed for two CDPRs of different sizes.

Many perturbations can exist in the system and all of them affect the system stability in a combined way. However, the sensitivity is a function of the perturbed parameter, as discussed in the previous section. Most importantly, the perturbation ranges within system stability scale with the CDPR geometry. 
To conclude, CDPRs with visual servoing control are robust to many perturbations, including modeling errors and MP pose estimation errors. Computation of the CSW is a good approach to evaluate this robustness.

\section{References}

1. E. Stump and V. Kumar, "Workspaces of cable-actuated parallel manipulators," Journal of Mechanical Design, vol. 128, no. 1, pp. 159-167, 2006.

2. R. Verhoeven, "Analysis of the Workspace of Tendon-Based," PhD thesis, Duisburg Gerhard Mercator University, 2004.

3. L. Gagliardini, "Discrete Reconfigurations of Cable-Driven Parallel Robots," PhD thesis, Ecole Centrale de Nantes, 2016.

4. S. Perreault, P. Cardou, C. M. Gosselin, and M. J.-D. Otis, "Geometric determination of the interference-free constant-orientation workspace of parallel cable-driven mechanisms," Journal of Mechanisms and Robotics, vol. 2, no. 3, 2010.

5. M. Arsenault, "Computation of the interference-free wrench feasible workspace of a 3-dof translational tensegrity robot," in Int. Conf on Cable-Driven Parallel Robots. Springer, 2019, pp. 185-196.

6. S. Lessanibahri, M. Gouttefarde, S. Caro, and P. Cardou, "Twist Feasibility Analysis of Cable-Driven Parallel Robots," in 3rd Int. Conf on Cable-Driven Parallel Robots, ser. Mechanisms and Machine Science, vol. 53, Québec, Canada, Aug. 2017, pp. 128-139.

7. G. Barrette and C. M. Gosselin, "Determination of the dynamic workspace of cabledriven planar parallel mechanisms," Journal of Mechanical Design, Transactions of the ASME, vol. 127, no. 2, pp. 242-248, 2005.

8. L. Gagliardini, M. Gouttefarde, and S. Caro, "Determination of a dynamic feasible workspace for cable-driven parallel robots," in Advances in Robot Kinematics 2016. Springer, 2018, vol. 4, pp. 361-370.

9. Z. Zake, F. Chaumette, N. Pedemonte, and S. Caro, "Vision-based control and stability analysis of a cable-driven parallel robot," IEEE Robotics and Automation Letters, vol. 4, no. 2, pp. 1029-1036, 2019.

10. Z. Zake, S. Caro, A. Suarez-Roos, F. Chaumette, and N. Pedemonte, "Stability analysis of pose-based visual servoing control of cable-driven parallel robots," Int. Conf on Cable-Driven Parallel Robots, CableCon 2019, pp. 73-84, 2019.

11. Z. Zake, F. Chaumette, N. Pedemonte, and S. Caro, "Robust 21/2d visual servoing of a cable-driven parallel robot thanks to trajectory tracking," IEEE Robotics and Automation Letters, vol. 5, no. 2, pp. 660-667, 2020.

12. F. Chaumette and S. Hutchinson, "Visual Servoing and visual tracking," in Handbook of Robotics, B. Siciliano and O. Khatib, Eds. Springer, 2008, pp. 563-583.

13. B. Siciliano, L. Sciavicco, L. Villani, and G. Oriolo, Robotics. Modelling, planning, control, M. Grimble and M. Johnson, Eds. Springer, 2010.

14. A. Pott, Cable-Driven Parallel Robots: Theory and Application. Springer, 2018.

15. H. K. Khalil, Nonlinear Systems, 2002.

16. L. Gagliardini, S. Caro, M. Gouttefarde, and A. Girin, "Discrete reconfiguration planning for Cable-Driven Parallel Robots," Mechanism and Machine Theory, vol. 100, pp. 313-337, 2016.

17. A. L. C. Ruiz, S. Caro, P. Cardou, and F. Guay, "ARACHNIS : Analysis of Robots Actuated by Cables with Handy and Neat Interface Software," in Cable-Driven Parallel Robots. Springer, 2015, pp. 293-305. 